\title{
Reliability based Generator Maintenance Scheduling using Integer Coded Differential Evolution Algorithm
}

\author{
G. Balaji \\ Assistant Professor \\ Department of Electrical Engg. \\ Annamalai University \\ Annamalai Nagar, India
}

\author{
R. Balamurugan, $\mathrm{PhD}$ \\ Assistant Professor \\ Department of Electrical Engg. \\ Annamalai University \\ Annamalai Nagar, India
}

\author{
L. Lakshminarasimman, \\ $\mathrm{PhD}$ \\ Associate Professor \\ Department of Electrical Engg. \\ Annamalai University \\ Annamalai Nagar, India
}

\begin{abstract}
In this paper, Generator Maintenance Scheduling (GMS) in a vertically integrated power system is considered. The objective of the GMS problem is to find the particular time interval for maintenance of power generating units with an intention of maximizing the security of the power system. In this paper, scheduling of generating units for planned preventive maintenance is formulated as a mixed integer optimization problem by considering maximizing the average value of reliability index subject to a set of nonlinear constraints. Integer Coded Differential Evolution (ICDE) algorithm is developed to solve the GMS problem. The Lagrange Multiplier method is used to find the overall production cost for the maintenance schedule that is obtained using ICDE algorithm. To demonstrate the effectiveness of the proposed approach, two test systems are considered and are validated by comparing results obtained with that of Integer Coded Particle Swarm Optimization. The test results reveal the capability of the proposed ICDE algorithm in finding optimal maintenance schedule for the generator maintenance scheduling problem.
\end{abstract}

\section{Keywords}

Generator Maintenance Scheduling, Reliability Maximization, Integer Coded Differential Evolution, Optimal Maintenance Schedule.

\section{INTRODUCTION}

Generator maintenance scheduling (GMS) is a complex combinatorial optimization problem and is a sub problem of integrated long term operations planning problem. During early periods, the installed capacity was more than the system demand such that schedule for maintenance of power generating units have been obtained without any difficulty. Due to the increase in power demand, there is a considerable increase in system size. The increase in the number of generating units and lower reserve margin makes the GMS more complex. The major intention of the GMS is to find the exact time period for starting of maintenance of power generating units with an objective of maximizing the reliability or minimizing the overall operational cost. Some of the earlier works used mathematical approaches like Dynamic Programming [DP] $[1,2]$, Branch and Bound [B \& B] technique [3] and Integer Programming [4] for solving GMS. Due to the presence of ambiguities, solving some systems for GMS using such mathematical approaches require excessive computational efforts. To obtain optimal or near optimal solution in a reasonable amount of time, artificial intelligent techniques have been proposed. The objective criterion of minimizing overall operational cost is considered and solved using Simulated Annealing [SA] [5], Genetic Algorithm [GA] [6], Tabu Search [TS] [7], Memetic Algorithm [MA] [8] and
Evolutionary Programming [EP] [9]. The reliability related with a power system is a measure of its ability to provide an adequate supply of electrical energy for the period of time. The reliability goal considered for solving the problem of maintenance scheduling of generating units is either deterministic or probabilistic. Schedule for maintenance of generators are obtained for the objective of level the reserve and TS is used for solving the problem [7]. The deterministic reliability criterion of minimizing sum of squares of reserve generation is considered in [10] and solved using novel GA which uses integer representation and fuzzy evaluation function. In [11], the meta-heuristic based hybrid approach is used to obtain the maintenance schedule for generators based on a reliability criterion. The reliability objective of level the reserve rate based maintenance schedule is obtained in [12] using code specific and constraint-transparent integral coded GA. GMS is considered in [13] in which all the objectives and constraints are fuzzified with the help of GA and is solved using fuzzy dynamic programming. Probabilistic reliability criterion of minimizing annual value of loss of load expectation is taken as objective of GMS problem and solution has been obtained using GA [14]. Loss of Load Probability is taken as objective in formulating GMS problem and has been solved using method of cumulants [15]. In this paper, Differential Evolution (DE) $[17,18]$ a powerful and successful evolutionary optimization algorithm is used for finding optimal solution for the GMS problem. The simple DE has the problem of struck at local optimum value [19]. If this problem is eradicated then it is possible to get a global optimum value. To alleviate the problem of premature convergence and to increase the diversity of population in the search space and to find near global optimal solution, DE highly depends on the population size. The problem like GMS has more number of decision variables which are continuous and integer. The continuous variables indicate the power output from committed generating units and integer decision variables denote the on/off status of power generating units. To obtain a near global optimum value for such high dimension problems, DE needs large number of population. This encourages the author to propose an algorithm for solving high dimensional GMS problem with considerable reduction in population size. In this paper, starting period for maintenance of power production units is considered as decision variables which are integers. In the proposed methodology, Integer Coded Differential Evolution (ICDE) is used as a main optimizer that optimizes the reliability objective so as to maximize the power system security and gives the reliability based maintenance schedule. Lagrange multiplier method is an iterative mathematical approach that is used to optimally dispatch the available generation that is obtained using DE based near global optimal maintenance schedule in order to meet the active power load demand. The 
use of this Lagrange multiplier method eliminates the use of continuous decision variables and also reduces the computation time required for optimizing the continuous variables considerably. The proposed methodology is validated by considering 4 and 22 units test system. The results are compared with that obtained through Integer coded particle swarm optimization (ICPSO) and Lagrange Multiplier method.

\section{NOMENCLATURE}

$t \quad$ - Time period index (week)

$T$ - Total number of sub periods (weeks) in the planning horizon

$i \quad$ - Power generating unit index

$N \quad$ - Total number of generators

$N C G \quad$ - Number of committed generators

$\omega \quad$ - Penalty factor

nc - Constraint index

NOC - Number of constraints

$\mathrm{CV}$ - Constraints violation

NI - Number of integers variables equal to number of units in the system

$P_{i}^{\min } \quad$ - Minimum limit generating unit $i$

$P_{i}^{\max } \quad$ - Maximum limit generating unit $i$

$R_{i} \quad$ - Rating of unit $i$

$P_{i t} \quad$ - Power output from generating unit $i$ in sub-period $t$

$D_{t} \quad$ - Active power load demand in sub-period $t$

$U_{i t} \quad-$ State variable equal to 1 if the unit $i$ in sub period $t$ is under maintenance and 0 otherwise

$E_{i} \quad$ - Earliest period in which maintenance of unit $i$ can start

$L_{i} \quad$ - Latest period in which maintenance of unit $i$ can start

$S_{i} \quad$ - Starting period of maintenance of unit $i \in\left[L_{i}, E_{i}\right]$

$M_{i} \quad$ - Maintenance duration of unit $i$

\section{PROBLEM FORMULATION}

The objective of maximizing reliability is as significant as that of minimizing cost. In this work, deterministic reliability objective criterion of maximizing the average value of reliability index in the given planning period is considered. The reliability index can be obtained by taking ratio between net reserve and gross reserve for each and every sub-period in the planning horizon. The net reserve is calculated as the difference between the total available generation, capacity of generators that are under maintenance and load demand. The gross reserve can be obtained by taking difference between the total available generation and load demand [16]. The reliability objective function is

$$
\operatorname{Max} \frac{1}{T} \sum_{t=1}^{T} R I(t)
$$

$$
\boldsymbol{R I}(t)=\frac{\sum_{i=1}^{N} P_{i}^{\max }-\sum_{i=1}^{N} P_{i}^{\max } U_{i t}-D_{t}}{\sum_{i=1}^{N} P_{i}^{\max }-D_{t}}
$$

The maintenance schedule obtained for the GMS problem should satisfy the following set of hard and soft constraints.

\subsection{Maintenance Window Constraint}

The planned preventive maintenance work of each generator present in the system must be scheduled and carried out between its earliest and latest period allowed for maintenance. This constraint ensures that once maintenance of the unit $i$ begins, the work have to be continued without any break off for the time period that is exactly equal to maintenance duration of unit $i$. The constraint can be expressed as

$$
U_{i t}=\left\{\begin{array}{lr}
1, & t=S_{i}, \ldots, S_{i}+M_{i}-1 \\
0, & \text { otherwise }
\end{array}\right.
$$

\subsection{Man Power Constraint}

It depends on the availability of man power for maintenance work. It guarantees that no two units can be maintained by the same maintenance crews. It is expressed in terms of $U_{i t}$ variables of the second unit $i 2$ as follows

$$
\sum_{t=S_{i 1}}^{S_{i 1}+M_{i 1}-1} U_{i 2, t}=0
$$

Equation (4), states that unit ' 2 ' should not be under maintenance when the duration on which unit ' 1 ' is under maintenance.

\subsection{Precedence Constraint}

In some occasions, some of the generators need maintenance to be completed prior to the starting of maintenance of other generating units. This is achieved with the help of precedence constraint. This constraint specifies the order in which maintenance on the generators has to be carried out. For instance, if maintenance of unit ' 1 ' ' is to be completed before the starting of maintenance of unit ' 2 ', then this constraint is given by

$$
S_{2}>S_{1}+M_{1}-1
$$

\subsection{4 Resource Constraint}

This constraint guarantees that the capacity on maintenance is less than the gross reserve at any time period in the planning horizon.

$$
\sum_{i=1}^{N} \boldsymbol{R}_{i} \boldsymbol{U}_{i t} \leq\left(\sum_{i=1}^{N} \boldsymbol{P}_{i}^{\max }-D_{t}\right)
$$

In this paper, it is assumed that the rating of unit $i$ is exactly equal to the maximum power that can be generated by unit $i$.

\subsection{Generator Limit Constraint}

The active power output from every power generating units must be within their lower and upper bounds. This is given by

$$
\boldsymbol{P}_{i}^{\min } \leq \boldsymbol{P}_{i t} \leq \boldsymbol{P}_{i}^{\max }
$$

where 


\subsection{Power Balance Constraint}

This constraint ensures that the total generation of all the units in every sub-period must be equal to the load demand on that sub-period $t$. This is given by

$$
\sum_{i=1}^{N} \boldsymbol{P}_{i t}=\boldsymbol{D}_{t}
$$

\section{PROPOSED METHODOLOGY}

Differential Evolution (DE) algorithm is simple evolutionary algorithm that creates offspring by combining parent individual and several other individuals of the same population $[17,18]$. An offspring replaces the parent if it has better fitness. The key steps involved in DE are initialization, mutation, crossover and selection. DE has been successfully applied to solve various difficult optimization problems and has been verified as a promising algorithm for solving real world optimization problems in diverse fields [20]. In this paper, the problem of maintenance scheduling of power producing units is considered with the perspective of maximizing the system security. The GMS problem is formulated as a mixed integer problem. Differential Evolution algorithm is suitably modified to handle the integer variables by including a rounding off operator. The integer variables denotes when the preventive maintenance of each generating units present in the system can begins. In this work, integer coded differential evolution (ICDE) is proposed for solving generator maintenance scheduling with the objective of maximizing the average value of reliability index subject to a set of constraints. The proposed ICDE algorithm for solving GMS has following steps

\subsection{Initialization}

The population of vector of integer variable that denotes the starting period of maintenance of each and every generator (X) is randomly initialized between its earliest and latest staring period as follows.

$$
\left(X_{i}^{k}\right)=\operatorname{round}\left(E_{i}^{k}+\operatorname{random}(0,1) \cdot\left(L_{i}^{k}-E_{i}^{k}\right)\right)
$$

where, $k=1,2, \ldots$, population size. The integer variables are checked for satisfying constraints (4) and (5). If any of the above constraint is violated for a generating unit, the integer variable is selected randomly between its earliest and latest starting period until the man power constraint and precedence constraint are satisfied and then the integer variables are checked for maintenance window constraint. After satisfying the constraints (3), (4), (5) and (6) the status of each unit $i$ is set at ' 1 ' from the starting period up to its corresponding maintenance duration period $\left(M_{i}\right)$ to denote that the unit $i$ is taken offline for planned preventive maintenance and ' 0 ' during other periods. The fitness function of the GMS problem to be maximized by ICDE is

$$
\psi=\left(1 /\left(\left(\frac{1}{\frac{1}{T} \sum_{t=1}^{T} \boldsymbol{R I}(\boldsymbol{t})}\right)+\sum_{n c=1}^{N O C} \omega_{n c}\left|\boldsymbol{C} \boldsymbol{V}_{n c}\right|\right)\right)
$$

\subsection{Mutation Phase}

The mutation phase is the second and most important phase in DE. The mutant integer vector or donor vector is produced in this phase. The most successful mutation strategies are given in [19]. The mutation strategy that is used in this work is $\mathrm{DE} / \mathrm{rand} / 1$. To create the mutant integer vector using $\mathrm{DE} / \mathrm{rand} / 1$, three vectors $r 1, r 2$ and $r 3$ are randomly selected from the population in the current generation $(G)$ and the weighted difference between two vectors is added with the third vector as follows

$$
\left(V_{k}^{G+1}\right)=\left(X_{r 1}^{G}+\operatorname{round}\left(F \cdot\left(X_{r 2}^{G}-X_{r 3}^{G}\right)\right)\right)
$$

The randomly chosen vectors $r 1, r 2$ and $r 3 \in[1, N P]$ which are mutually different and is also different from base index $k$. the scaling or mutation factor $F \in[0.1,1]$. The scaling factor $F$ ensures the fastest possible convergence. The obtained donor vector is checked in order to satisfy man power and precedence constraint. If any of the constraint is violated for a unit, the integer variable is selected randomly between its earliest and latest starting period until constraints (4) and (5) are satisfied and then the integer variables are checked for maintenance window constraint and resource constraint. The perturbed individual $\boldsymbol{V}_{\boldsymbol{k}}^{\boldsymbol{G}+1}$ thus obtained is a mutated integer vector for its parent integer vector $\boldsymbol{X}_{\boldsymbol{k}}^{\boldsymbol{G}}$.

\subsection{Crossover (or) Recombination Phase}

After completing the mutation phase, recombination phase is used to create offspring or trial vector for each parent or target vector. The offspring $\boldsymbol{U}_{\boldsymbol{k}}^{\boldsymbol{G}+1}$ is created by applying uniform binomial crossover operation to each pair of parent integer vector $\boldsymbol{X}_{\boldsymbol{k}}^{\boldsymbol{G}}$ and its corresponding mutant integer vector $\boldsymbol{V}_{\boldsymbol{k}}^{\boldsymbol{G + 1}}$ as follows

$$
U_{h k}^{G+1}= \begin{cases}V_{h k}^{G+1}, & \text { random }_{k}(0,1) \leq \boldsymbol{C R} \\ \boldsymbol{X}_{h k}^{G}, & \text { otherwise }\end{cases}
$$

where $h=1,2, \ldots, N I$ and crossover factor $C R \in[0,1]$. The binomial crossover operator copies the $h^{\text {th }}$ parameter of the mutant vector to the corresponding element in the trial vector if random number is less than or equal to $C R$. Otherwise, it is copied from the corresponding target vector. The individual gene in the trial integer vector represents the starting period of each generating unit. The status of each unit $i$ is set at ' 1 ' from the starting period up to its corresponding maintenance duration period and ' 0 ' during other periods. Then using equation (10), the fitness function is evaluated.

\subsection{Selection Phase}

In this phase, the population of target or parent vectors $\boldsymbol{X}_{\boldsymbol{k}}^{\boldsymbol{G}+1}$ is generated for the successive generation. The parent vectors for next generation are imitated from the trial integer vector if it has better fitness function value than its corresponding parent vector if not it is reproduced from parent integer vector as follows

$$
\boldsymbol{X}_{k}^{G+1}= \begin{cases}U_{k}^{G+1}, & \text { if } \psi\left(U_{k}^{G+1}\right) \geq \psi\left(X_{k}^{G}\right) \\ X_{k}^{G}, & \text { otherwise }\end{cases}
$$

After finding out the near global optimum solution for the GMS problem that confirms maximum system security using integer coded differential evolution algorithm, the Lagrange Multiplier method is used to optimally dispatch the available generation in order to meet the load demand with minimum production cost. The Lagrange Multiplier approach has the following steps.

Step 1: With an initial value of $\lambda$, power output of each committed generator $\left(P_{i}\right)$ in sub period $t$ is calculated using 


$$
\boldsymbol{P}_{i t}=\frac{\lambda-\boldsymbol{b}_{i}}{2 \boldsymbol{c}_{i}}
$$

The generated output power is checked for satisfying constraint (7). If it is not so, set at its maximum value if it exceeds the maximum limit of that generator or at its minimum value if it is lesser than the minimum limit of that generator.

Step 2: The change in power output can be calculated using

$$
\Delta \boldsymbol{P}^{K}=\boldsymbol{D}_{t}-\sum_{i=1}^{N C G} \boldsymbol{P}_{i t}
$$

Step 3: The new value of $\lambda$ for next iteration is calculated by adding change in $\lambda$ with previous value of $\lambda$ as follows

$$
\begin{aligned}
\Delta \lambda^{K} & =\frac{\Delta \boldsymbol{P}^{K}}{\sum_{i=1}^{N C G} \frac{1}{2 c_{i}}} \\
\lambda^{K+1} & =\lambda^{K}+\Delta \lambda^{K}
\end{aligned}
$$

The steps (14) - (17) are repeated until $\Delta \mathrm{P}$ is zero. Thus optimal generation of committed generating units with minimum production cost can be found in order to meet the load demand in sub-period $t$, thus satisfying constraint (8).

\section{SIMULATION RESULTS AND DISCUSSION}

In the proposed work, the reliability based generator maintenance scheduling is considered in order to maximize the system security. The reliability objective criterion of

maximizing the average value of reliability index is considered subject to various hard and soft constraints. Integer coded differential evolution algorithm is proposed for solving GMS problem so as to obtain near global optimum solution. The Lagrange Multiplier method is used to economically dispatch the existing power generation to meet the weekly load demand. To demonstrate the proposed approach, two test systems 4 units and 22 units are considered [7]. The effectiveness of the proposed algorithm is authenticated by comparing the simulation results with that obtained using Integer coded particle swarm optimization (ICPSO). The program is developed using MATLAB 7.7 on a personal computer with $2 \mathrm{GHz}$ Core 2 Duo CPU.

\subsection{Test System 1: 4 Units system}

A small test system having 4 power producing units that should be maintained within a planning period of 8 weeks. There are 4 integer variables that indicate the starting period of 4 generating units. Due to precedence constraint, the maintenance of unit 1 should be completed before the starting of the maintenance of unit 2. Due to man power constraint, unit 1 and unit 2 should not be maintained simultaneously. To compare the results obtained, the population size for 4 unit system is fixed at 10 and the maximum number of generations is set at 1000 for both ICDE and ICPSO algorithms. The generator data are taken from [7]. The generator data are given in table 1 .

The results of both the algorithms are studied for the following two cases.

Case 1: Load demand in every week is considered as it is in literature [7].

Case 2: Total load demand (Sum of all weekly load demand) is increased by $15 \%$.

Since the test systems are taken from [7], the results are also compared with that obtained in [7] for case 1 alone.

5.1.1 Case 1: For case 1, the maintenance schedule obtained by ICDE and ICPSO for maximizing security of the system is shown in table 2 . Since test system 1 is a small system, both the algorithms produce the same maintenance schedule. The value of control parameters of ICDE that gives near optimal maintenance schedule are $\mathrm{F}=0.70$ and $\mathrm{CR}=0.90$ whereas the value of that of ICPSO are $\mathrm{C} 1=\mathrm{C} 2=2.0$ and inertia weight $\mathrm{W}=0.45$.

In table 2, ' 1 ' indicates that the generating unit is under maintenance and ' 0 ' point outs that the generator is under running condition. In table 2 , it can be seen that all the generating units are under maintenance for the specified maintenance duration without any interruption and are not under maintenance repetitively in the planning period of 8 weeks. From table 2, it is clear that unit 1 and 2 are not taken offline for preventive maintenance in the same period thus satisfying manpower constraint. Also, the maintenance of unit 1 gets completed before the commencement of maintenance of unit 2 satisfying precedence constraint. In every week, the capacity on maintenance is less than the gross reserve thereby satisfying resource constraint as shown in figure 1. Most of the units are under maintenance during low load period as shown in figure 1 so that the reliability index is high. Table 3 shows the generation schedule obtained using Lagrange Multiplier method.

The maximum average value of reliability index for 4 units test system is 0.5588 and the production cost for the above maintenance schedule is \$ 3400434.56 . For the maintenance schedule of 4 unit system that is obtained through new tabu search algorithm in [7], for level the reserve objective, the average value of reliability index is found to be 0.5513 and for the same schedule, Lagrange Multiplier approach gives the production cost of $\$ 3393095.33$ whereas for another objective of minimizing the total generator operating cost, they have found the maintenance schedule for which the

\begin{tabular}{|c|c|c|c|c|}
\hline Units (I) & 1 & 2 & 3 & 4 \\
\hline Maintenance Duration: $M_{i}$ (Weeks) & 4 & 2 & 2 & 1 \\
\hline $\mathbf{P}_{\text {Min. }}(\mathbf{M W})$ & 0 & 0 & 0 & 0 \\
\hline $\mathbf{P}_{\text {Max. }}(\mathbf{M W})$ & 200 & 200 & 300 & 90 \\
\hline Variable O\&M Cost: $\mathrm{b}_{\mathrm{OM}}(\$ / \mathrm{MWh})$ & 0.2 & 0.2 & 0.4 & 0.5 \\
\hline Fuel Cost: $a_{1}(\$ / h)$ & 78 & 80 & 110 & 60 \\
\hline Fuel Cost: $b_{1}(\$ / M W h)$ & 7.97 & 7.80 & 7.65 & 8.40 \\
\hline
\end{tabular}
average value of reliability index is found to be 0.5534 and the production cost is found to be $\$ 3392728.13$.

Table 1: Generator Data of 4 units system 


\begin{tabular}{l|c|c|c|c}
\hline Fuel Cost: $\mathbf{c}_{\mathbf{1}}\left(\mathbf{\$} / \mathbf{M W}^{\mathbf{2}} / \mathbf{h}\right)$ & 0.00482 & 0.00462 & 0.00465 & 0.00610 \\
Cost: $\mathbf{a}=\mathbf{a}_{\mathbf{1}} \mathbf{( \$ / \mathbf { h } )}$ & 78 & 80 & 110 & 60 \\
Cost: $\mathbf{b}=\mathbf{b}_{\mathbf{1}}+\mathbf{b}_{\mathrm{OM}}(\mathbf{\$} / \mathbf{M W h})$ & 8.17 & 8.00 & 8.05 & 8.90 \\
Cost: $\left.\mathbf{c}=\mathbf{c}_{\mathbf{1}} \mathbf{( \$ \mathbf { M W }} \mathbf{/ h}\right)$ & 0.00482 & 0.00462 & 0.00465 & 0.00610 \\
\hline
\end{tabular}

Table 2: Maintenance Schedule for 4 units system for case 1

\begin{tabular}{c|c|c|c|c|c|c|c|c}
\hline Unit/Week & $\mathbf{1}$ & $\mathbf{2}$ & $\mathbf{3}$ & $\mathbf{4}$ & $\mathbf{5}$ & $\mathbf{6}$ & $\mathbf{7}$ & $\mathbf{8}$ \\
\hline $\mathbf{1}$ & $\mathbf{1}$ & $\mathbf{1}$ & $\mathbf{1}$ & $\mathbf{1}$ & 0 & 0 & 0 & 0 \\
$\mathbf{2}$ & 0 & 0 & 0 & 0 & 0 & $\mathbf{1}$ & $\mathbf{1}$ & 0 \\
$\mathbf{3}$ & 0 & 0 & 0 & 0 & 0 & 0 & $\mathbf{1}$ & $\mathbf{1}$ \\
$\mathbf{4}$ & 0 & 0 & 0 & 0 & 0 & 0 & $\mathbf{1}$ & 0 \\
\hline
\end{tabular}

Table 3: Generation Schedule of $\mathbf{4}$ units system for case 1

\begin{tabular}{c|c|c|c|c|c|c|c|c}
\hline Unit/Week & $\mathbf{1}$ & $\mathbf{2}$ & $\mathbf{3}$ & $\mathbf{4}$ & $\mathbf{5}$ & $\mathbf{6}$ & $\mathbf{7}$ & $\mathbf{8}$ \\
\hline $\mathbf{1}$ & $\mathbf{0}$ & $\mathbf{0}$ & $\mathbf{0}$ & $\mathbf{0}$ & 72.96 & 125.21 & 187 & 118.74 \\
$\mathbf{2}$ & 119.29 & 125.10 & 129.10 & 130.19 & 94.51 & $\mathbf{0}$ & $\mathbf{0}$ & 142.28 \\
$\mathbf{3}$ & 113.14 & 118.92 & 122.89 & 123.98 & 88.53 & 142.69 & $\mathbf{0}$ & $\mathbf{0}$ \\
$\mathbf{4}$ & 16.57 & 20.98 & 24.01 & 24.83 & 0 & 39.10 & $\mathbf{0}$ & 33.98 \\
\hline
\end{tabular}

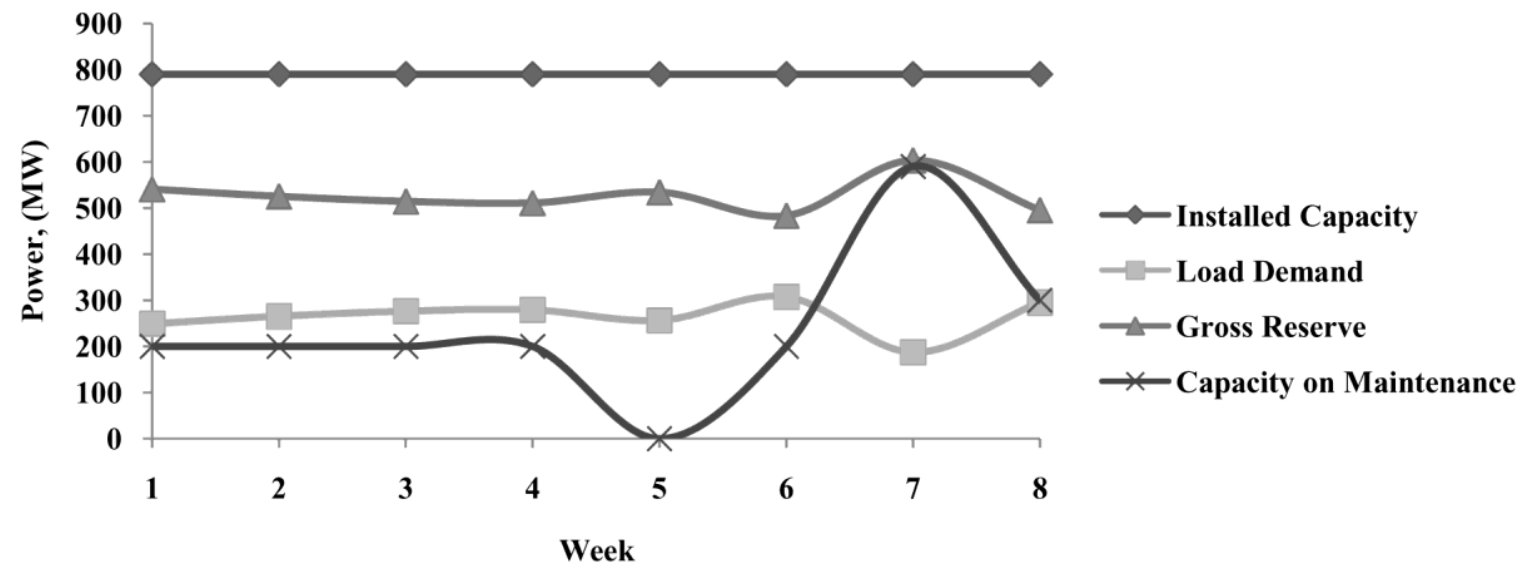

Figure 1: Capacity on Maintenance for case 1

\subsubsection{Case 2:}

In case 2, the sum of all weekly load demand given in [7] is increased by $15 \%$ and is distributed randomly to each period. The load data is given in Figure 2.

For case 2, the maintenance schedule based on maximum system security obtained by ICDE and ICPSO and generation schedule obtained by Lagrange Multiplier approach are given in table 4.

From table 4, it is clear that the maintenance of unit 1 gets completed before the beginning of maintenance of unit 2 satisfying precedence constraint. Also, it can be seen that unit
1 and 2 are not taken offline for preventive maintenance simultaneously thus satisfying manpower constraint. Figure 3 clearly indicates that in every week, the capacity on maintenance is less than the gross reserve thereby satisfying resource constraint. The value of control parameters are $\mathrm{F}=$ 0.7 and CR $=0.9$ in case of ICDE and that is for ICPSO are $\mathrm{C} 1=\mathrm{C} 2=2.0$ and inertia weight $\mathrm{W}=0.45$.

The average value of reliability index obtained by both the algorithms is 0.5181 . Since in case 2, the load is increased, the reliability index gets reduced. The production cost for case 2 is found to be $\$ 3894089.06$. 


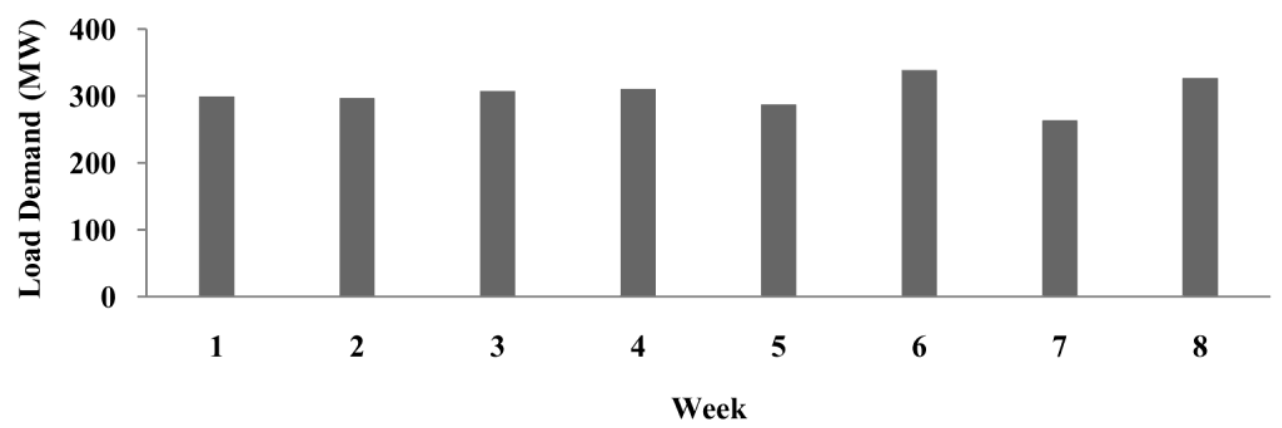

Figure 2: Weekly Peak Load Demand of 4 Units System for case 2

Table 4: Maintenance Schedule and Generation Schedule of 4 units system for case 2

\begin{tabular}{c|c|c|c|c|c|c|c|c}
\hline Unit/Week & $\mathbf{1}$ & $\mathbf{2}$ & $\mathbf{3}$ & $\mathbf{4}$ & $\mathbf{5}$ & $\mathbf{6}$ & $\mathbf{7}$ & $\mathbf{8}$ \\
\hline $\mathbf{1}$ & 84.63 & $\mathbf{0}$ & $\mathbf{0}$ & $\mathbf{0}$ & $\mathbf{0}$ & 136.53 & 180.90 & 130.03 \\
$\mathbf{2}$ & 106.70 & 136.73 & 140.73 & 141.82 & 147.16 & $\mathbf{0}$ & $\mathbf{0}$ & 154.06 \\
$\mathbf{3}$ & 100.64 & 130.47 & 134.44 & 135.53 & 140.84 & 154.42 & $\mathbf{0}$ & $\mathbf{0}$ \\
$\mathbf{4}$ & 7.03 & 29.81 & 32.82 & 33.64 & $\mathbf{0}$ & 48.05 & 83.10 & 42.91 \\
\hline
\end{tabular}

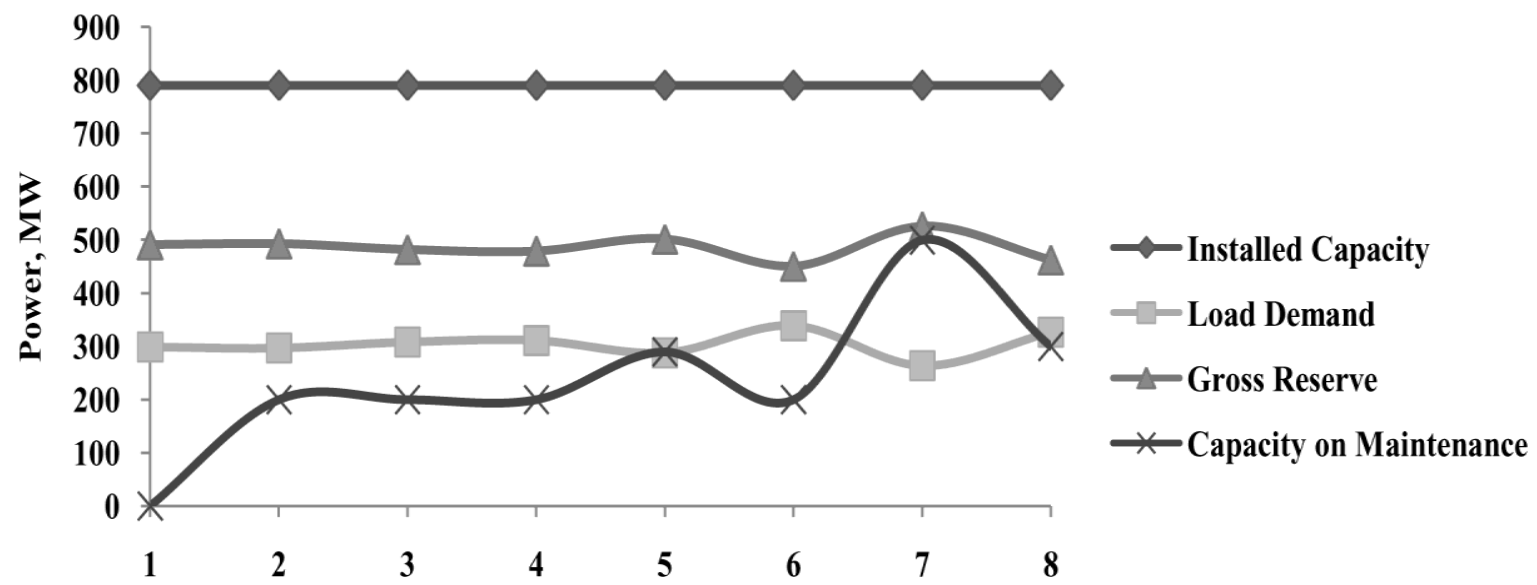

Week

Figure 3: Capacity on Maintenance for case 2

\subsection{Test System 2: 22 Units system}

The second test system is a real size large scale system having 22 units for which the maintenance work should be completed within the planning horizon of one year (52 weeks). The generator data is taken from [7] and is given table 5.

Due to man power constraints, units 15 and 16, units 21 and 22 should not be maintained at the same time period. Due to precedence constraints, maintenance of units 2 and units 5 have to be completed first before the commencement of maintenance of units 3 and units 6 respectively. To compare the results obtained, the population size for 22 unit system is fixed at 50 and the maximum number of generations is set at 3000 for both ICDE and ICPSO algorithms.

\subsubsection{Case 1}

The weekly load pattern is taken as it is in [7]. The weekly load data is given in figure 4.

For maximum reliability for case 1 , the maintenance schedule obtained using proposed ICDE and ICPSO are given in table 6 and 7 respectively. In table 6 and 7, it can be seen that both algorithms satisfy man power constraint and precedence constraint. It table 6, it can be seen that most of the generating units are taken off for preventive maintenance during light load conditions. Figure 5 shows the capacity on maintenance in every week of the planning horizon. In figure 5 , it can be seen that the capacity on maintenance in every week is less than its corresponding gross reserve thereby satisfying resource constraint. 
Table 5: Generator Data of 22 units test system

\begin{tabular}{|c|c|c|c|c|c|c|c|c|c|c|}
\hline \multirow{2}{*}{$\begin{array}{l}\text { Unit } \\
\text { (i) }\end{array}$} & \multirow{2}{*}{$\mathbf{M}_{\mathbf{i}}$} & \multirow{2}{*}{$\begin{array}{l}\mathbf{P}_{\text {Min. }} \\
\text { (MW) }\end{array}$} & \multirow{2}{*}{$\begin{array}{l}\mathbf{P}_{\text {Max. }} \\
\text { (MW) }\end{array}$} & \multirow{2}{*}{$\begin{array}{c}\text { Variable } \\
\text { O\&M } \\
\text { Cost: } \mathbf{b}_{\mathrm{OM}}\end{array}$} & \multicolumn{3}{|c|}{ Fuel Cost } & \multicolumn{3}{|c|}{ Cost } \\
\hline & & & & & $\mathbf{a}_{1}$ & $b_{1}$ & $\mathbf{c}_{1}$ & $\mathbf{a}=\mathbf{a}_{1}(\$ / \mathbf{h})$ & $\begin{array}{c}\mathbf{b}=\mathbf{b}_{1}+\mathbf{b}_{\mathrm{OM}} \\
(\$ / \mathbf{M W h})\end{array}$ & $\begin{array}{c}c=c_{1} \\
\left(\$ / M^{2} / h\right)\end{array}$ \\
\hline 1 & 6 & 0 & 100 & 0.25 & 70 & 8.00 & 0.00585 & 70 & 8.25 & 0.00585 \\
\hline 2 & 3 & 0 & 100 & 0.20 & 70 & 8.00 & 0.00580 & 70 & 8.20 & 0.00580 \\
\hline 3 & 3 & 0 & 100 & 0.20 & 70 & 8.00 & 0.00580 & 70 & 8.20 & 0.00580 \\
\hline 4 & 3 & 0 & 100 & 0.20 & 70 & 8.00 & 0.00580 & 70 & 8.20 & 0.00580 \\
\hline 5 & 6 & 0 & 90 & 0.35 & 60 & 8.00 & 0.00610 & 60 & 8.35 & 0.00610 \\
\hline 6 & 4 & 0 & 90 & 0.30 & 60 & 8.00 & 0.00610 & 60 & 8.30 & 0.00610 \\
\hline 7 & 3 & 0 & 95 & 0.20 & 68 & 8.00 & 0.00579 & 68 & 8.20 & 0.00579 \\
\hline 8 & 4 & 0 & 100 & 0.20 & 72 & 8.00 & 0.00565 & 72 & 8.20 & 0.00565 \\
\hline 9 & 5 & 0 & 650 & 0.52 & 525 & 7.00 & 0.00120 & 525 & 7.52 & 0.00120 \\
\hline 10 & 12 & 0 & 610 & 0.50 & 510 & 7.20 & 0.00142 & 510 & 7.70 & 0.00142 \\
\hline 11 & 4 & 0 & 91 & 0.20 & 62 & 8.25 & 0.00600 & 62 & 8.45 & 0.00600 \\
\hline 12 & 8 & 0 & 100 & 0.30 & 74 & 8.15 & 0.00578 & 74 & 8.45 & 0.00578 \\
\hline 13 & 3 & 0 & 100 & 0.20 & 70 & 8.00 & 0.00580 & 70 & 8.20 & 0.00580 \\
\hline 14 & 6 & 0 & 100 & 0.25 & 70 & 8.00 & 0.00585 & 70 & 8.25 & 0.00585 \\
\hline 15 & 5 & 0 & 220 & 0.25 & 85 & 7.90 & 0.00460 & 85 & 8.15 & 0.00460 \\
\hline 16 & 6 & 0 & 220 & 0.25 & 87 & 7.95 & 0.00464 & 87 & 8.20 & 0.00464 \\
\hline 17 & 5 & 0 & 100 & 0.20 & 69 & 8.18 & 0.00570 & 69 & 8.38 & 0.00570 \\
\hline 18 & 5 & 0 & 100 & 0.25 & 69 & 8.17 & 0.00572 & 69 & 8.42 & 0.00572 \\
\hline 19 & 3 & 0 & 220 & 0.25 & 81 & 7.90 & 0.00463 & 81 & 8.15 & 0.00463 \\
\hline 20 & 3 & 0 & 220 & 0.25 & 82 & 7.95 & 0.00462 & 82 & 8.20 & 0.00462 \\
\hline 21 & 3 & 0 & 240 & 0.30 & 82 & 7.40 & 0.00410 & 82 & 7.70 & 0.00410 \\
\hline 22 & 5 & 0 & 240 & 0.30 & 80 & 7.42 & 0.00415 & 80 & 7.72 & 0.00415 \\
\hline
\end{tabular}

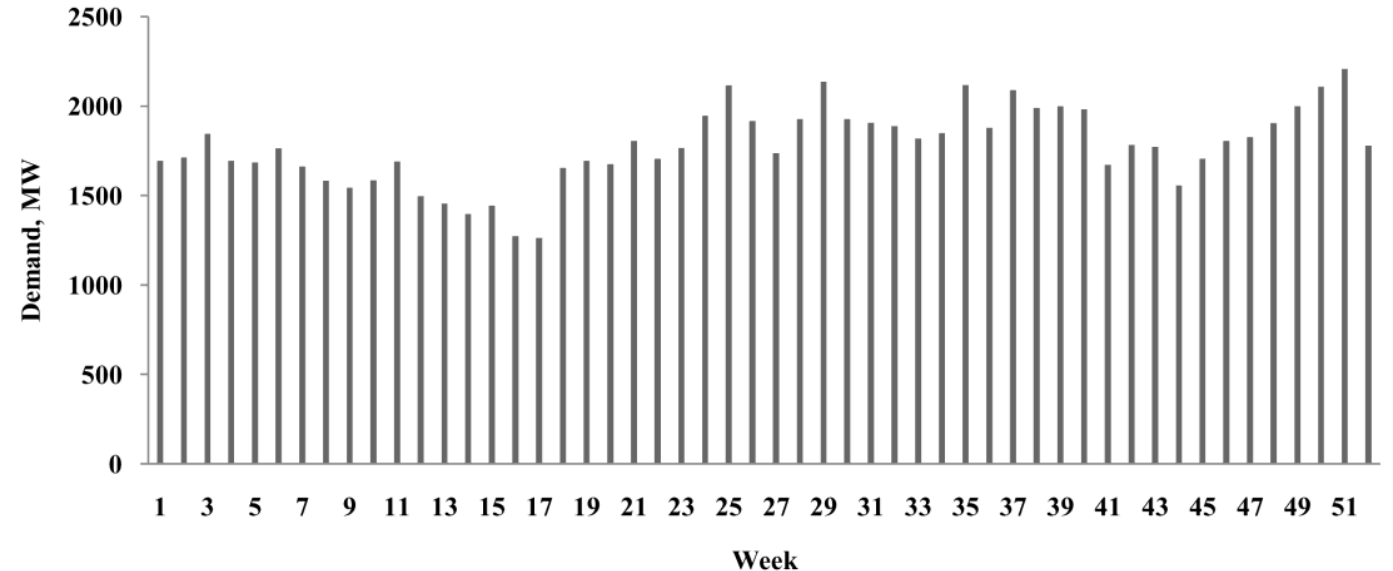

Figure 4: Weekly Peak Load Profile of 22 Units System 


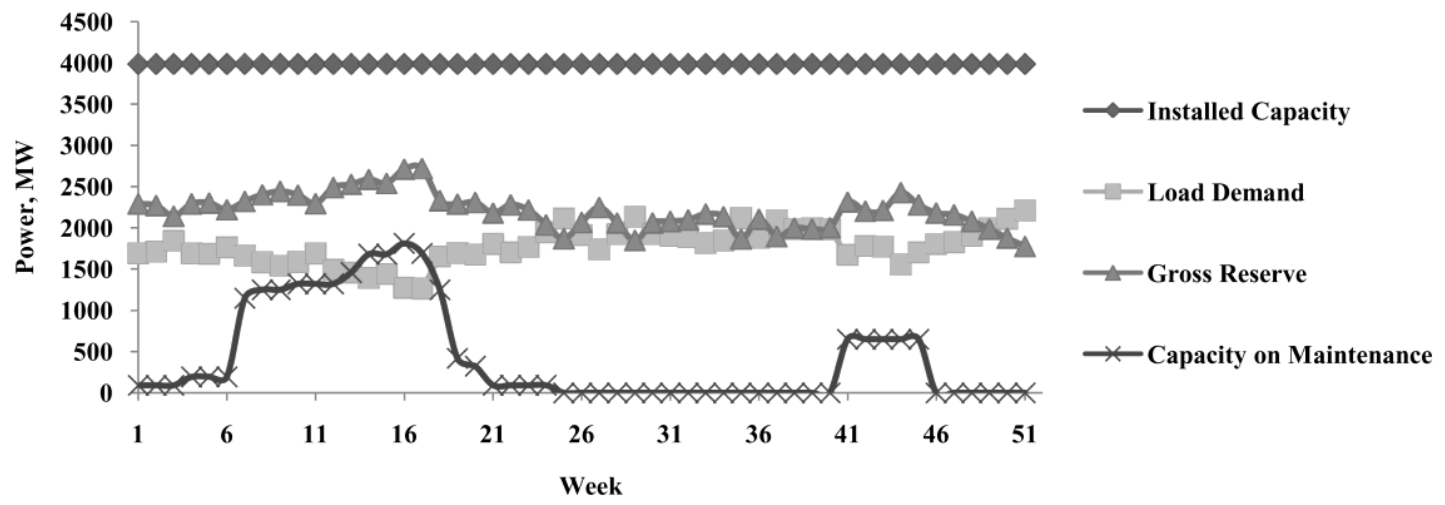

Figure 5: Capacity on Maintenance for case 1

The maximum average value of reliability index obtained for 22 unit system for case 1 using ICDE is 0.8235 and the production cost that is found using Lagrange Multiplier method is $\$ 148624224.65$. The tuning parameters are $\mathrm{F}=0.5$ and $\mathrm{CR}=0.93$. While using ICPSO, 0.8229 is the maximum average value of reliability index that is found and corresponding overall operation cost that includes production cost and variable operation and maintenance cost is found to be $\$ 148693524.20$. The tuning parameters are $\mathrm{C} 1=\mathrm{C} 2=2$ and inertia weight is equal to 0.58 .

For the maintenance schedule of 22 unit system that is obtained in [7], for level the reserve objective, the average value of reliability index is found to be 0.8114 and for the same schedule, Lagrange Multiplier approach gives the production cost of $\$ 148652977.99$ whereas for the objective of minimizing the total generator operating cost, they have found the maintenance schedule for which the average value of reliability index is found to be 0.8132 and the production cost is found to be $\$ 148705625.34$.

5.2.2 Case 2: In case 2, sum of weekly load demand profile shown in figure 4 is increased by $15 \%$ and is distributed randomly between every time period. The load data of 22 units system for second case is given in figure 6. For case 2, similar to case 1 , it is considered that due to man power constraints, units 15 and 16, units 21 and 22 should not be maintained at the same time period. Due to precedence constraints, maintenance of units 2 and units 5 have to be completed first before the commencement of maintenance of units 3 and units 6 respectively.

Table 6: Maintenance Schedule (ICDE)

\begin{tabular}{c|c|c}
\hline Weeks & Demand & Units on Maintenance \\
\hline $\mathbf{1}$ & 1694 & 11 \\
$\mathbf{2}$ & 1714 & 11 \\
$\mathbf{3}$ & 1844 & 11 \\
$\mathbf{4}$ & 1694 & 4,11 \\
$\mathbf{5}$ & 1684 & 4,7 \\
$\mathbf{6}$ & 1763 & 4,7 \\
$\mathbf{7}$ & 1663 & $1,7,10,12,21$ \\
$\mathbf{8}$ & 1583 & $1,10,12,14,18,21$ \\
$\mathbf{9}$ & 1543 & $1,10,12,14,18,21$ \\
$\mathbf{1 0}$ & 1586 & $1,5,10,12,14,15,18$ \\
$\mathbf{1 1}$ & 1690 & $1,5,10,12,14,15,18$ \\
$\mathbf{1 2}$ & 1496 & $1,5,10,12,14,15,18$ \\
$\mathbf{1 3}$ & 1456 & $5,10,12,14,15,17,22$ \\
\hline
\end{tabular}

\begin{tabular}{|c|c|c|}
\hline 14 & 1396 & $2,5,10,12,15,17,20,22$ \\
\hline 15 & 1443 & $2,5,10,13,16,17,20,22$ \\
\hline 16 & 1273 & $2,10,13,16,17,19,20,22$ \\
\hline 17 & 1263 & $3,8,10,13,16,17,19,22$ \\
\hline 18 & 1655 & $3,8,10,16,19$ \\
\hline 19 & 1695 & $3,8,16$ \\
\hline 20 & 1675 & 8,16 \\
\hline 21 & 1805 & 6 \\
\hline 22 & 1705 & 6 \\
\hline 23 & 1766 & 6 \\
\hline 24 & 1946 & 6 \\
\hline 25 & 2116 & $* * * * *$ \\
\hline 26 & 1916 & $* * * * *$ \\
\hline 27 & 1737 & $* * * * *$ \\
\hline 28 & 1927 & $* * * * *$ \\
\hline 29 & 2137 & $* * * * *$ \\
\hline 30 & 1927 & $* * * * *$ \\
\hline 31 & 1907 & $* * * * *$ \\
\hline 32 & 1888 & $* * * * *$ \\
\hline 33 & 1818 & $* * * * *$ \\
\hline 34 & 1848 & $* * * * *$ \\
\hline 35 & 2118 & $* * * * *$ \\
\hline 36 & 1879 & $* * * * *$ \\
\hline 37 & 2089 & $* * * * *$ \\
\hline 38 & 1989 & $* * * * *$ \\
\hline 39 & 1999 & $* * * * *$ \\
\hline 40 & 1982 & $* * * * *$ \\
\hline 41 & 1672 & 9 \\
\hline 42 & 1782 & 9 \\
\hline 43 & 1772 & 9 \\
\hline 44 & 1556 & 9 \\
\hline 45 & 1706 & 9 \\
\hline 46 & 1806 & $* * * * *$ \\
\hline 47 & 1826 & $* * * * *$ \\
\hline 48 & 1906 & $* * * * *$ \\
\hline 49 & 1999 & $* * * * *$ \\
\hline 50 & 2109 & $* * * * *$ \\
\hline 51 & 2209 & $* * * * *$ \\
\hline 52 & 1779 & $* * * * *$ \\
\hline
\end{tabular}


Table 7: Maintenance Schedule (ICPSO)

\begin{tabular}{|c|c|c|}
\hline Weeks & Demand & Units on Maintenance \\
\hline 1 & 1694 & 12 \\
\hline 2 & 1714 & 12 \\
\hline 3 & 1844 & 12 \\
\hline 4 & 1694 & 12 \\
\hline 5 & 1684 & 12 \\
\hline 6 & 1763 & 10,12 \\
\hline 7 & 1663 & $10,12,15$ \\
\hline 8 & 1583 & $5,10,12,15$ \\
\hline 9 & 1543 & $5,10,15$ \\
\hline 10 & 1586 & $5,10,15$ \\
\hline 11 & 1690 & $5,10,15$ \\
\hline 12 & 1496 & $1,5,10,16$ \\
\hline 13 & 1456 & $1,5,10,16,17,18$ \\
\hline 14 & 1396 & $1,6,10,16,17,18$ \\
\hline 15 & 1443 & $1,2,6,7,10,13,16,17,18,20,21$ \\
\hline 16 & 1273 & $1,2,6,7,10,13,16,17,18,20,21$ \\
\hline 17 & 1263 & $1,2,6,7,10,13,16,17,18,20,21$ \\
\hline 18 & 1655 & $* * * * *$ \\
\hline 19 & 1695 & $* * * * *$ \\
\hline 20 & 1675 & $* * * * *$ \\
\hline 21 & 1805 & $* * * * *$ \\
\hline 22 & 1705 & $* * * * *$ \\
\hline 23 & 1766 & $* * * * *$ \\
\hline 24 & 1946 & $* * * * *$ \\
\hline 25 & 2116 & $* * * * *$ \\
\hline 26 & 1916 & $* * * * *$ \\
\hline 27 & 1737 & $* * * * *$ \\
\hline
\end{tabular}

\begin{tabular}{l|l|c}
\hline $\mathbf{2 8}$ & 1927 & $* * * * *$ \\
$\mathbf{2 9}$ & 2137 & $* * * * *$ \\
$\mathbf{3 0}$ & 1927 & $* * * * *$ \\
$\mathbf{3 1}$ & 1907 & $* * * * *$ \\
$\mathbf{3 2}$ & 1888 & $* * * * *$ \\
$\mathbf{3 3}$ & 1818 & $* * * * *$ \\
$\mathbf{3 4}$ & 1848 & $* * * * *$ \\
$\mathbf{3 5}$ & 2118 & $* * * * *$ \\
$\mathbf{3 6}$ & 1879 & $* * * * *$ \\
$\mathbf{3 7}$ & 2089 & $* * * * *$ \\
$\mathbf{3 8}$ & 1989 & $* * * * *$ \\
$\mathbf{3 9}$ & 1999 & $* * * * *$ \\
$\mathbf{4 0}$ & 1982 & $* * * * *$ \\
$\mathbf{4 1}$ & 1672 & $8,9,11,14,22$ \\
$\mathbf{4 2}$ & 1782 & $8,9,11,14,22$ \\
$\mathbf{4 3}$ & 1772 & $3,4,8,9,11,14,19,22$ \\
$\mathbf{4 4}$ & 1556 & $3,4,8,9,11,14,19,22$ \\
$\mathbf{4 5}$ & 1706 & $3,4,9,14,19,22$ \\
$\mathbf{4 6}$ & 1806 & 14 \\
$\mathbf{4 7}$ & 1826 & $* * * * *$ \\
$\mathbf{4 8}$ & 1906 & $* * * * *$ \\
$\mathbf{4 9}$ & 1999 & $* * * * *$ \\
$\mathbf{5 0}$ & 2109 & $* * * * *$ \\
$\mathbf{5 1}$ & 2209 & $* * * * *$ \\
$\mathbf{5 2}$ & 1779 & $* * * * *$ \\
\hline & &
\end{tabular}

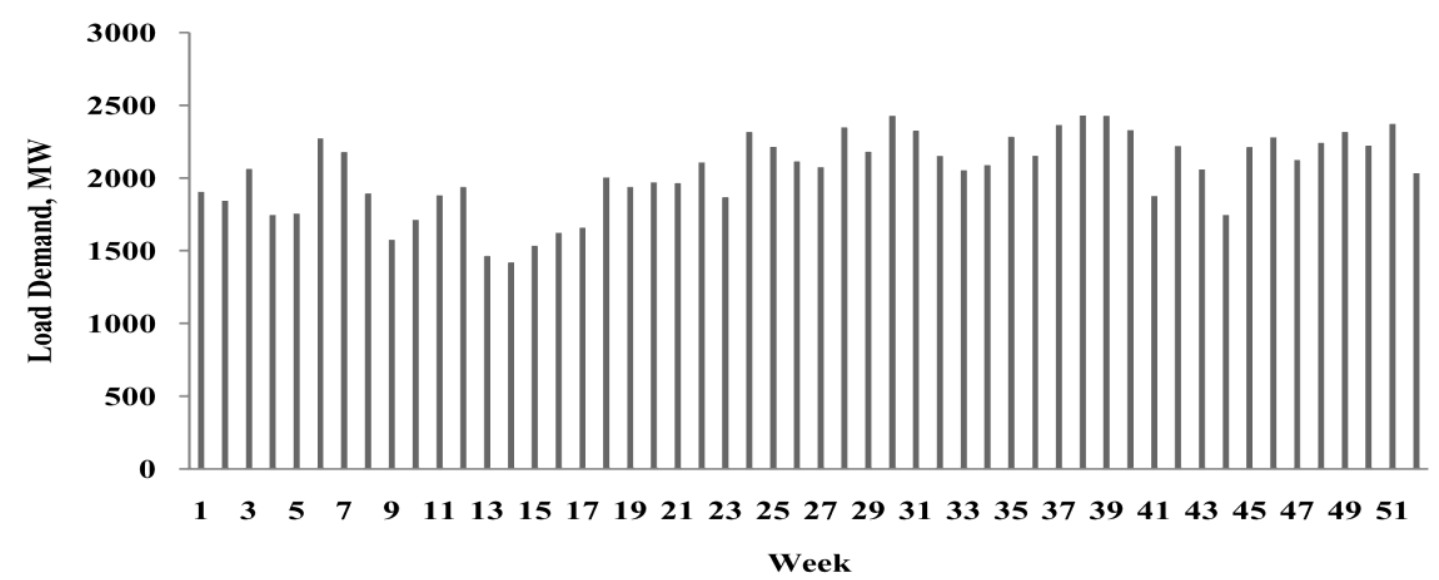

Figure 6: Weekly Load Demand Profile for 22 Units System for case

The maintenance schedule obtained using ICDE and ICPSO are given in table 8 and table 9 respectively. The tuning parameters of ICDE algorithm are $\mathrm{F}=0.5$ and $\mathrm{CR}=0.91$ and that of ICPSO algorithm are $\mathrm{C} 1=\mathrm{C} 2=2$ and inertia weight $\mathrm{W}=0.46$. The beginning of planned preventive maintenance work of unit 3 and unit 6 have been initiated only after the finishing of maintenance of unit 2 and unit 5 and hence both algorithms satisfies precedence constraints as shown in table 8 and 9. Similarly unit 15 and 16 and unit 21 and 22 are not under maintenance simultaneously; thereby manpower constraint is satisfied by ICDE and ICPSO algorithms.
The capacity on maintenance is shown in figure 7 . It is evident that the capacities on maintenance in each and every time period in the planning horizon are lesser than the gross reserve thus satisfying resource constraint. The comparison of results obtained for 22 units using ICDE and ICPSO in terms of average value of reliability index and its associated overall operation cost found using Lagrange Multiplier method is given in table 10. From the table, it can be concluded that ICDE gives better maintenance schedule for maximizing the system security. In both the cases, most of the units are under maintenance during low load period thereby ICDE gives near 


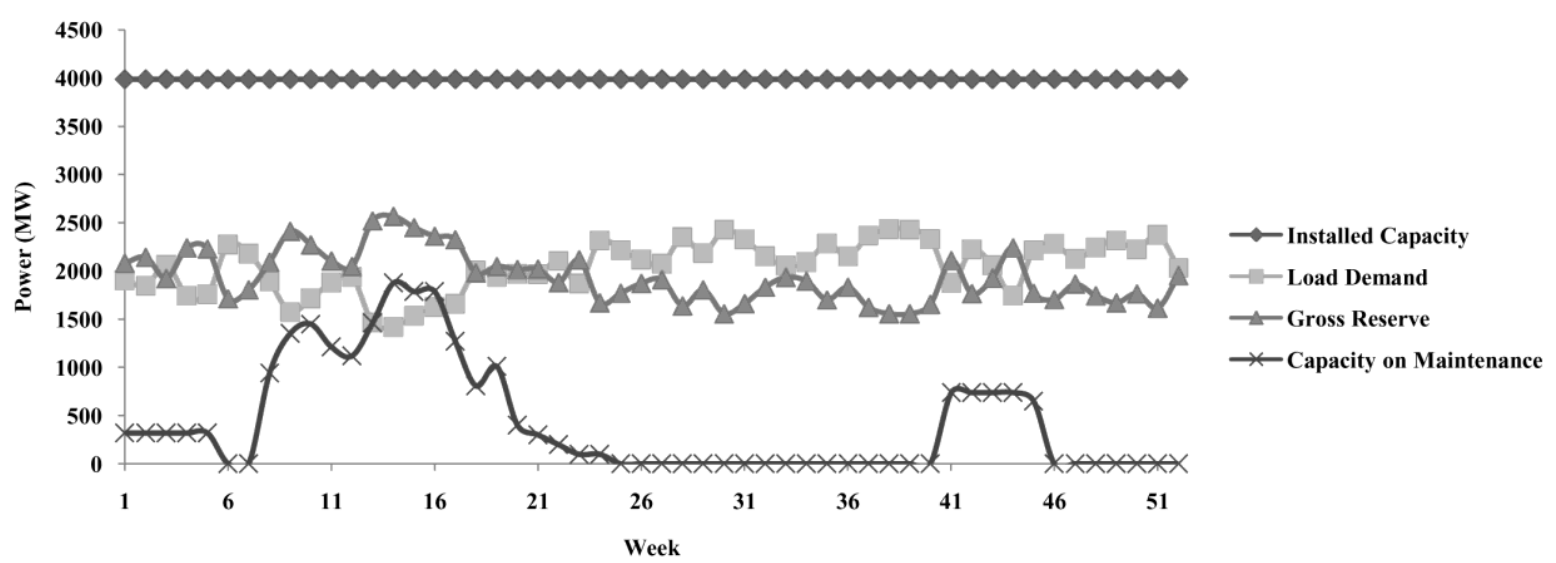

Figure 7: Capacity on Maintenance for case 2

Table 8: Maintenance Schedule (ICDE)

\begin{tabular}{|c|c|c|}
\hline Weeks & Demand & Units on Maintenance \\
\hline 1 & 1904 & 15,17 \\
\hline 2 & 1844 & 15,17 \\
\hline 3 & 2062 & 15,17 \\
\hline 4 & 1746 & 15,17 \\
\hline 5 & 1755 & 15,17 \\
\hline 6 & 2271 & $* * * * *$ \\
\hline 7 & 2179 & $* * * * *$ \\
\hline 8 & 1893 & $10,11,21$ \\
\hline 9 & 1575 & $2,5,10,11,19,21$ \\
\hline 10 & 1713 & $2,5,10,11,12,19,21$ \\
\hline 11 & 1881 & $2,5,10,11,12,19$ \\
\hline 12 & 1939 & $5,10,12,16,18$ \\
\hline 13 & 1464 & $5,10,12,14,16,18,22$ \\
\hline 14 & 1419 & $5,7,10,12,13,14,16,18,20,22$ \\
\hline 15 & 1534 & $7,10,12,13,14,16,18,20,22$ \\
\hline 16 & 1623 & $7,10,12,13,14,16,18,20,22$ \\
\hline 17 & 1658 & $10,12,14,16,22$ \\
\hline 18 & 2004 & $3,10,14$ \\
\hline 19 & 1938 & $1,3,4,8,10$ \\
\hline 20 & 1970 & $1,3,4,8$ \\
\hline 21 & 1965 & $1,4,8$ \\
\hline 22 & 2107 & 1,8 \\
\hline 23 & 1868 & 1 \\
\hline 24 & 2316 & 1 \\
\hline 25 & 2215 & $* * * * *$ \\
\hline 26 & 2115 & $* * * * *$ \\
\hline 27 & 2074 & $* * * * *$ \\
\hline 28 & 2348 & $* * * * *$ \\
\hline 29 & 2181 & $* * * * *$ \\
\hline 30 & 2428 & $* * * * *$ \\
\hline 31 & 2325 & $* * * * *$ \\
\hline 32 & 2151 & $* * * * *$ \\
\hline 33 & 2053 & $* * * * *$ \\
\hline 34 & 2089 & $* * * * *$ \\
\hline
\end{tabular}

\begin{tabular}{l|l|c}
\hline $\mathbf{3 5}$ & 2283 & $* * * * *$ \\
$\mathbf{3 6}$ & 2153 & $* * * * *$ \\
$\mathbf{3 7}$ & 2364 & $* * * * *$ \\
$\mathbf{3 8}$ & 2430 & $* * * * *$ \\
$\mathbf{3 9}$ & 2428 & $* * * * *$ \\
$\mathbf{4 0}$ & 2330 & $* * * * *$ \\
$\mathbf{4 1}$ & 1876 & 6,9 \\
$\mathbf{4 2}$ & 2220 & 6,9 \\
$\mathbf{4 3}$ & 2059 & 6,9 \\
$\mathbf{4 4}$ & 1745 & 6,9 \\
$\mathbf{4 5}$ & 2213 & 9 \\
$\mathbf{4 6}$ & 2279 & $* * * * *$ \\
$\mathbf{4 7}$ & 2123 & $* * * * *$ \\
$\mathbf{4 8}$ & 2242 & $* * * * *$ \\
$\mathbf{4 9}$ & 2316 & $* * * * *$ \\
$\mathbf{5 0}$ & 2221 & $* * * * *$ \\
$\mathbf{5 1}$ & 2371 & $* * * * *$ \\
$\mathbf{5 2}$ & 2033 & $* * * * *$ \\
\hline
\end{tabular}

Table 9: Maintenance Schedule (ICPSO)

\begin{tabular}{c|c|c}
\hline Weeks & Demand & Units on Maintenance \\
\hline $\mathbf{1}$ & 1904 & $5,15,18,19$ \\
$\mathbf{2}$ & 1844 & $5,15,18,19$ \\
$\mathbf{3}$ & 2062 & $5,15,18,19$ \\
$\mathbf{4}$ & 1746 & $5,15,18$ \\
$\mathbf{5}$ & 1755 & $5,15,18$ \\
$\mathbf{6}$ & 2271 & 5 \\
$\mathbf{7}$ & 2179 & $* * * * *$ \\
$\mathbf{8}$ & 1893 & 10 \\
$\mathbf{9}$ & 1575 & $1,2,10,12,21$ \\
$\mathbf{1 0}$ & 1713 & $1,2,10,12,14,21$ \\
$\mathbf{1 1}$ & 1881 & $1,2,10,12,14,21$ \\
$\mathbf{1 2}$ & 1939 & $1,10,12,14,16$ \\
$\mathbf{1 3}$ & 1464 & $1,6,8,10,11,12,14,16,20,22$ \\
$\mathbf{1 4}$ & 1419 & $1,3,6,8,10,11,12,14,16,20,22$ \\
$\mathbf{1 5}$ & 1534 & $3,6,8,10,11,12,14,16,20,22$ \\
\hline
\end{tabular}




\begin{tabular}{|c|c|c|}
\hline 16 & 1623 & $3,6,8,10,11,12,16,22$ \\
\hline 17 & 1658 & $10,16,22$ \\
\hline 18 & 2004 & 10 \\
\hline 19 & 1938 & 10 \\
\hline 20 & 1970 & $* * * * *$ \\
\hline 21 & 1965 & $* * * * *$ \\
\hline 22 & 2107 & $* * * * *$ \\
\hline 23 & 1868 & $* * * * *$ \\
\hline 24 & 2316 & $* * * * *$ \\
\hline 25 & 2215 & $* * * * *$ \\
\hline 26 & 2115 & $* * * * *$ \\
\hline 27 & 2074 & $* * * * *$ \\
\hline 28 & 2348 & $* * * * *$ \\
\hline 29 & 2181 & $* * * * *$ \\
\hline 30 & 2428 & $* * * * *$ \\
\hline 31 & 2325 & $* * * * *$ \\
\hline 32 & 2151 & $4,7,9$ \\
\hline 33 & 2053 & $4,7,9$ \\
\hline 34 & 2089 & $4,7,9$ \\
\hline 35 & 2283 & 9 \\
\hline 36 & 2153 & 9 \\
\hline 37 & 2364 & $* * * * *$ \\
\hline 38 & 2430 & $* * * * *$ \\
\hline 39 & 2428 & $* * * * *$ \\
\hline 40 & 2330 & $* * * * *$ \\
\hline 41 & 1876 & 17 \\
\hline 42 & 2220 & 13,17 \\
\hline 43 & 2059 & 13,17 \\
\hline 44 & 1745 & 13,17 \\
\hline 45 & 2213 & 17 \\
\hline 46 & 2279 & $* * * * *$ \\
\hline 47 & 2123 & $* * * * *$ \\
\hline 48 & 2242 & $* * * * *$ \\
\hline 49 & 2316 & $* * * * *$ \\
\hline 50 & 2221 & $* * * * *$ \\
\hline 51 & 2371 & $* * * * *$ \\
\hline 52 & 2033 & $* * * * *$ \\
\hline
\end{tabular}

Table 10: Comparison of results for 22 units system for case 2

\begin{tabular}{c|c|c}
\hline Algorithm & $\begin{array}{c}\text { Average value of } \\
\text { Reliability Index }\end{array}$ & $\begin{array}{c}\text { Overall Operation } \\
\text { Cost }\end{array}$ \\
\hline ICDE & 0.8043 & $\$ 169221692.04$ \\
ICPSO & 0.8021 & $\$ 169247623.92$ \\
\hline
\end{tabular}

From the results, it can be seen that for both the cases of test systems 1 and 2, ICDE gives the maintenance schedule for same control parameters which proves the consistency of the algorithm.

\section{CONCLUSION}

In this paper, the problem of maintenance scheduling of power generating units based on maximizing the reliability of the system is considered. The problem is subjected to different equality and inequality constraints. The solutions for reliability based GMS problems are obtained using the integer coded differential evolution algorithm. Lagrange Multiplier method is used to economically dispatch the available generation for the maintenance schedule that is attained using ICDE in order to get minimum overall operational cost. The proposed algorithm is validated by considering two test systems; a small and a real size system. The numerical results prove that ICDE algorithm is effective in finding better maintenance schedule in terms of maximum reliability when compared to integer coded particle swarm optimization approach. The future scope of this work is to find a optimal maintenance schedule for GMS problem that must be a best compromise solution between cost and reliability.

\section{ACKNOWLEDGMENTS}

The authors gratefully acknowledge the support and facilities provided by the authorities of Annamalai University, Annamalainagar, India to carry out this research work.

\section{REFERENCES}

[1] H. H. Zurn, V. H. Quintana, "Generator maintenance scheduling via successive approximations dynamic programming", IEEE Transactions on Power Apparatus and Systems, 94(2); (1975); 665 - 671.

[2] Zia. A. Yamayee, K. Sidenblad, M. Yoshimura, "A Computationally Efficient Optimal Maintenance Scheduling Method", IEEE Transactions on Power Apparatus and Systems, 102(2); (1983); 330 - 338.

[3] G. T. Egan, T. S. Dillon, K. Morsztyn, “An Experimental method of determination of Optimal Maintenance Schedules in Power Systems using the Branch and Bound technique", IEEE Transactions on Systems, Man and Cybernetics, 6( 8); (1976); 538 - 547.

[4] J.F. Dopazo, H.M. Merrill, "Optimal Maintenance Scheduling using Integer Programming", IEEE Transactions on Power Apparatus and Systems, 94(5); (1975); $1537-1545$

[5] T. Satoh, K. Nara, "Maintenance Scheduling by using Simulated Annealing Method", IEEE Transactions on Power Systems, 6(5); (1991); 850 - 857.

[6] S. Baskar, P. Subbaraj, M.V.C Rao, S. Tamilselvi, "Genetic algorithms solution to generator maintenance scheduling with modified genetic operators", IEE Proceedings on Generation, Transmission, Distribution, 150(1); (2003); $56-60$.

[7] I. El - Amin, S. Duffuaa, M. Abbas, "A Tabu search algorithm for maintenance scheduling of generating units", Electric Power System Research, 54; (2000); 91 99.

[8] E. K. Burke, A.J. Smith, "Hybrid Evolutionary Techniques for the Maintenance Scheduling Problem", IEEE Transactions on Power Systems, 15(1); (2000); 122 -128 .

[9] M. Y. El-Sharkh, A.A. El-Keib, H. Chen, "A fuzzy evolutionary programming-based solution methodology for security-constrained generation maintenance scheduling", Electric Power System Research, 67; (2003); $67-72$.

[10] K.P. Dahal, C.J. Aldridge, J.R. McDonald, "Generator maintenance scheduling using a genetic algorithm with a fuzzy evaluation function", Fuzzy Sets and System, 102; (1999); $21-29$. 
[11] K.P. Dahal, N. Chakpitak, "Generator maintenance scheduling in power systems using meta heuristic-based hybrid approaches", Electric Power System Research, 77; (2007); $771-779$.

[12] Y. Wang, E. Handschin, "A new genetic algorithm for preventive unit maintenance scheduling of power systems", Electrical Power and Energy Systems, 22; (2000); $343-348$.

[13] S.J. Huang, "Generator maintenance scheduling: a fuzzy system approach with genetic enhancement", Electric Power Systems Research, 4l; (1997); 233 - 239.

[14] A. Volkanovski, B. Mavko, T. Bosevski, A. Causevski, M. Cepin, "Genetic algorithm optimization of the maintenance scheduling of generating units in a power system", Reliability Engineering and System Safety, 93; (2008); $757-767$.

[15] J.P. Stremel, "Maintenance Scheduling For Generation System Planning", IEEE Transactions on Power Apparatus and Systems, 100(3); (1981); 1410 - 1419.
[16] A.J. Conejo, R.G. Bertrand, M.D. Salazar, "Generation Maintenance Scheduling in Restructured Power Systems", IEEE Transactions on Power Systems, 20 (2): (2005); $984-992$.

[17] R. Storn, K. V. Price, "Differential evolution - A simple and efficient heuristic for global optimization over continuous spaces", Journal of Global Optimization, 11; (1997); $341-359$.

[18] R. Storn, K. V. Price, "Minimizing the real function of the ICEC'96 contest by differential evolution", Proceedings of IEEE Conference on Evolutionary Computation, Nagoya, Japan, (1996); 842-844.

[19] S. Das, A. Abraham, U.K. Chakraborty, A. Konar, "Differential Evolution Using a Neighborhood-Based Mutation Operator", IEEE Transactions On Evolutionary Computation, 13(3); (2009); 526 - 553.

[20] A. K. Qin, V. L. Huang, P. N. Suganthan, "Differential Evolution Algorithm With Strategy Adaptation for Global Numerical Optimization", IEEE Transactions On Evolutionary Computation, 13(2); (2009); 398 - 417 